\title{
Numerical and Experimental Approaches on the Motion of a Tethered System
}

\author{
Shoichiro TAKEHARA ${ }^{* *}$, Yoshiaki TERUMICHI ${ }^{* * *}$, Masahiro NOHMI ${ }^{* * * *}$ \\ and Kiyoshi SOGABE ${ }^{* * *}$ \\ **Tokyo Metropolitan University, 1-1 Minami-Ohsawa, Hachioji, Tokyo, 192-0397, Japan \\ E-mail: stakeha@tmu.ac.jp \\ ***Sophia University, 7-1, Kioi-cho, Chiyoda-ku, Tokyo, 102-8554, Japan \\ E-mail: y-terumi@sophia.ac.jp \\ ${ }^{\star \star \star \star K}$ Kagawa University, Hayashi-cho, Takamatsu, Kagawa, 761-0396, Japan \\ E-mail: nohmi@eng.kagawa-u.ac.jp
}

\begin{abstract}
In the present paper, the motion of a tethered system with large deformation and large displacement is discussed. In general, a tether is a cable or a wire rope, and a tethered system consists of a tether and the equipment attached to the tether. A tethered subsatellite in space is an example of a tethered system. In the present study, a tethered system consisting of a very flexible body (the tether) and a rigid body at one end is considered as the analytical model. A flexible body in planer motion is described using the Absolute Nodal Coordinate Formulation. Using this formulation, the motion of a flexible body with large deformation, rotation and translation can be expressed with the accuracy of rigid body motion. The combination of the flexible body motion and the rigid body motion is performed, and their interaction is discussed.

Experiments are performed to investigate the fundamental motion of the tethered system and to evaluate the validity of the numerical formulation. Experiments were conducted using a steel tether and a rubber tether in gravity space. In addition, an experiment of the motion of the tethered system with a rigid body in microgravity space was conducted.
\end{abstract}

Key words: Tethered System, Multibody Dynamics, Microgravity Experiment, Flexible Body, Large Deformation and Displacement, Coupled Motion

\section{Introduction}

Applications of tethered systems with equipment at one end have various possibilities for engineering in extreme environmental conditions, for example, in space or under deep sea. Tethered subsatellites ${ }^{(1)(2)}$ and tethered robots ${ }^{(3)}$ in space are examples of such systems. In order to simplify analysis, models in which the motion of tethered objects is considered as being equivalent to the motion of a pendulum have been often adopted for tethered systems. Systems composed of a string with small deformation and a mass system have also been considered $^{(4)(5)}$.

The tension of the tether has a large influence on the motion of the tether, even if the tension is small, because there is little influence of gravitational force acting in a constant direction in extreme environments such as in space or under deep sea. In such circumstances, the motion of the tether often has large rotation and deformation. Moreover, it is assumed that the motion of the tethered system is complex because the coupling motion 
between the tether and the attached equipment is excited. Therefore, the tethered system should be modeled as a flexible body attached to a rigid body.

Numerical approaches for the motion of the tethered system with large rotation and deformation have been proposed. In the present paper, Absolute Nodal Coordinate Formulation $^{(6)-(8)}$ is adapted to the tether motion. The constraint due to the combination of the tether and a rigid body is described. The augment formulation is applied in the numerical simulation.

On the other hand, experimental approaches on the motion of the tethered system have been almost nonexistent ${ }^{(9)}$. We attempted to perform two types of experiments. The first type was the tether motion with large rotation, deformation and translation. Two types of materials, steel and rubber, were used in these experiments. These experiments were performed under gravity. In the other type of experiment, the motion of the system consisting of a tether and a rigid body was observed under microgravity.

From these experimental results, the characteristics of the motion of the tether and the tethered system are clarified. These results lead to an explanation of the mechanism of the coupling motion between a tether and a rigid body with large rotation, deformation and translation. Furthermore, these experimental results are in good agreement with the numerical results. Therefore, the proposed method for the modeling and the formulation of the tethered system can be considered to be efficient.

\section{Analytical model and formulation}

\subsection{Analytical model}

In the present paper, the tethered system of a flexible body and a rigid body is considered, as shown in Fig. 1. The motion of the system is constrained in plane. In order to solve the large deformation problem, the flexible body is formulated using the Absolute Nodal Coordinate Formulation (ANCF). The augment formulation is applied for the formulation of the tethered system. In the present paper, the fundamental motion of the interaction between the tether and the rigid body is considered.

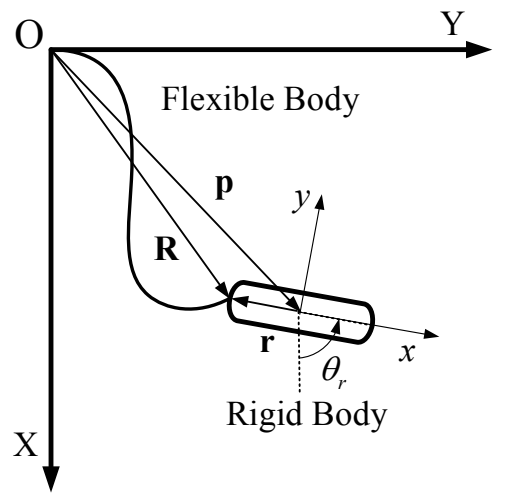

Fig. 1 Analytical model

\subsection{Formulation of motion of system with rigid body}

In this section, the motion of the tethered system is formulated. First, we explain the outline of the ANCF, which was proposed by A. A. Shabana et $\mathrm{al}^{(6)-(8)}$. This formulation can express the motion of a flexible body with large rotation, displacement and deformation. In this formulation, global coordinates and position vector gradients are used as the nodal coordinates. One element located in the absolute coordinates $O-X Y Z$ is shown in Fig. 2. The initial configuration and current configuration are designated $O A$ and $O^{\prime} A^{\prime}$, respectively. The global position vector $\mathbf{r}$ of an arbitrary point $x^{\prime}$ on the element is defined in terms of the nodal coordinates and the shape function 


$$
\mathbf{r}=\left[\begin{array}{ll}
r_{1} & r_{2}
\end{array}\right]^{T}=\mathbf{S e},
$$

where $\mathbf{S}$ is the shape function.

The following shape function is used:

$$
\mathbf{S}=\left[\begin{array}{cc}
1-3 \xi^{2}+2 \xi^{3} & 0 \\
0 & 1-3 \xi^{2}+2 \xi^{3} \\
l_{e}\left(\xi-2 \xi^{2}+\xi^{3}\right) & 0 \\
0 & l_{e}\left(\xi-2 \xi^{2}+\xi^{3}\right) \\
3 \xi^{2}-2 \xi^{3} & 0 \\
0 & 3 \xi^{2}-2 \xi^{3} \\
l_{e}\left(\xi^{3}-\xi^{2}\right) & 0 \\
0 & l_{e}\left(\xi^{3}-\xi^{2}\right)
\end{array}\right]^{T}
$$

where $\xi=x / l_{e}, x$ is the coordinate along the body axis in the initial configuration, $l_{e}$ is the length of the element. The vector of element nodal coordinates is

$$
\mathbf{e}=\left[\begin{array}{llllllll}
e_{1} & e_{2} & e_{3} & e_{4} & e_{5} & e_{6} & e_{7} & e_{8}
\end{array}\right]^{T},
$$

where $e_{1}, e_{2}, e_{5}$ and $e_{6}$ are the positions of nodes $O^{\prime}$ and $A^{\prime}$, and $e_{3}, e_{4}, e_{7}$, and $e_{8}$ are the slopes of the element nodes at nodes $O^{\prime}$ and $A^{\prime}$.

Using global position vector $\mathbf{r}$, the kinetic energy for one element $T$ is represented as

$$
T=\frac{1}{2} \int_{V} \rho \dot{\mathbf{r}}^{T} \dot{\mathbf{r}} d V=\frac{1}{2} \dot{\mathbf{e}}^{T} \mathbf{M}_{a} \dot{\mathbf{e}}
$$

where $\rho$ and $V$ are the density of the tether and the volume of the element, respectively, and $\mathbf{M}_{a}$ is the mass matrix of one element and is defined as

$$
\mathbf{M}_{a}=\int_{V} \rho \mathbf{S}^{T} \mathbf{S} d V .
$$

In order to obtain the elastic force, we selected the element coordinate system. A deformed element is shown in Fig. 3. If the point $O$ on the element is selected as the reference point, the position vector of an arbitrary point with respect to point $O$ can be written as

$$
\mathbf{u}=\left[\begin{array}{ll}
u_{x} & u_{y}
\end{array}\right]^{T}=\left[\begin{array}{c}
\left(\mathbf{S}_{1}-\mathbf{S}_{1 O}\right) \mathbf{e} \\
\left(\mathbf{S}_{\mathbf{2}}-\mathbf{S}_{\mathbf{2} O}\right) \mathbf{e}
\end{array}\right],
$$

where $\mathbf{S}_{1}$ and $\mathbf{S}_{2}$ are the rows of the element shape function matrix, and $\mathbf{S}_{1 O}$ and $\mathbf{S}_{2 O}$ are the rows of the shape function matrix defined at the reference point $O$. The unit vectors $\mathbf{i}$ and $\mathbf{j}$ are defined as follows:

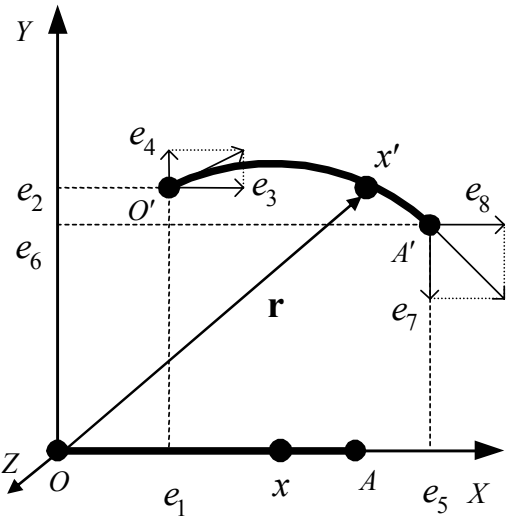

Fig. 2 Element of the flexible body in the ANCF

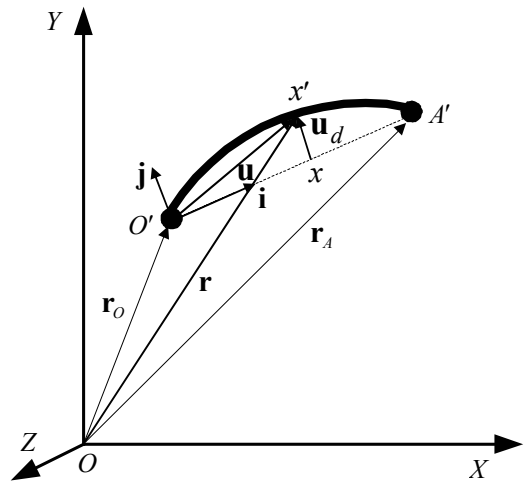

Fig. 3 Deformed element 


$$
\begin{aligned}
& \mathbf{i}=\left[\begin{array}{ll}
i_{x} & i_{y}
\end{array}\right]^{T}=\frac{\mathbf{r}_{A}-\mathbf{r}_{0}}{\left|\mathbf{r}_{A}-\mathbf{r}_{0}\right|}, \\
& \mathbf{j}=\left[\begin{array}{ll}
j_{x} & j_{y}
\end{array}\right]^{T}=\mathbf{k} \times \mathbf{i},
\end{aligned}
$$

where $\mathbf{i}$ is a unit vector along the selected element axis, $\mathbf{j}$ is a unit vector perpendicular to $\mathbf{i}$, and $\mathbf{k}$ is a unit vector along the $Z$ axis. Using this vector, the longitudinal and transverse displacements of the element can be written as follows:

$$
\mathbf{u}_{d}=\left[\begin{array}{l}
u_{l} \\
u_{t}
\end{array}\right]=\left[\begin{array}{c}
\mathbf{u}^{T} \mathbf{i}-x \\
\mathbf{u}^{T} \mathbf{j}
\end{array}\right]=\left[\begin{array}{c}
u_{x} i_{x}+u_{y} i_{y}-x \\
u_{x} j_{x}+u_{y} j_{y}
\end{array}\right] .
$$

Using the above expressions, the elastic potential energy of one element $U$ is represented as

$$
U=\frac{1}{2} \int_{0}^{l_{e}}\left(E a\left(\frac{\partial u_{l}}{\partial x}\right)^{2}+E I\left(\frac{\partial^{2} u_{t}}{\partial x^{2}}\right)^{2}\right) d x,
$$

where $E$ is the modulus of elasticity and $I$ is the second moment of area.

The stiffness vector $\mathbf{Q}_{\mathbf{k}}$ can be defined in terms of the strain energy $U$ as

$$
\mathbf{Q}_{\mathbf{k}}=-\left(\frac{\partial U}{\partial \mathbf{e}}\right) \text {. }
$$

The equation of motion of one element can be written as

$\mathbf{M}_{a} \ddot{\mathbf{e}}=\mathbf{Q}_{k}+\mathbf{Q}_{a}$,

where $\mathbf{Q}_{a}$ contains the generalized external forces including gravity.

The equations of motion of the tether can then be written as

$\mathbf{M}_{t} \ddot{\mathbf{e}}_{t}=\mathbf{Q}_{t}$,

where $\mathbf{M}_{t}$ is the mass matrix, $\mathbf{e}_{t} \equiv\left[\begin{array}{llll}e_{1} & e_{2} & \cdots & e_{4(n+1)}\end{array}\right]^{T}$ is the absolute nodal coordinate, and $\mathbf{Q}_{t} \equiv\left[\begin{array}{llll}Q_{1} & Q_{2} & \cdots & Q_{4(n+1)}\end{array}\right]^{T}$ consists of the external force and the elastic force of all elements of the tether. $n$ is the number of the element.

This formulation is extended to the motion of the flexible body with a rigid body. The equation of motion of the rigid body is

$$
\mathbf{M}_{r} \ddot{\mathbf{q}}_{r}=\mathbf{Q}_{r},
$$

where $\mathbf{M}_{r}=\operatorname{diag}\left[\begin{array}{lll}m_{r} & m_{r} & I_{r}\end{array}\right]$ is the mass matrix of the rigid body, $m_{r}$ is the mass of the rigid body, and $I_{r}$ is the inertia moment of the rigid body.

$\mathbf{q}_{r}=\left[\begin{array}{lll}x_{r} & y_{r} & \theta_{r}\end{array}\right]^{T}$ is the general coordinate of the rigid body. The coordinates $x_{r}$ and $y_{r}$ denote the center of mass $\left(m_{r}\right)$ of the rigid body. $\theta_{r}$ is the rotation of the rigid body.

$\mathbf{Q}_{r}=\left[\begin{array}{lll}F x_{r} & F y_{r} & M_{r}\end{array}\right]^{T}$ consists of the external force and the moment of the rigid body.

The equation of motion of the tethered system is

$\mathbf{M} \ddot{\mathbf{q}}=\mathbf{Q}$,

where $\mathbf{q}=\left[\begin{array}{ll}\mathbf{e}_{t}^{T} & \mathbf{q}_{r}^{T}\end{array}\right]^{T}, \mathbf{Q}=\left[\begin{array}{ll}\mathbf{Q}_{t}^{T} & \mathbf{Q}_{r}^{T}\end{array}\right]^{T}, \mathbf{M}=\left[\begin{array}{cc}\mathbf{M}_{t} & \mathbf{0} \\ \mathbf{0} & \mathbf{M}_{r}\end{array}\right]$.

In Fig.1, $\mathbf{R}=\left[\begin{array}{ll}e_{4 n+1} & e_{4 n+2}\end{array}\right]^{T}$ is the position vector of the connecting position between the bottom end of the tether and the upper end of the rigid body, $e_{4 n+1}$ and $e_{4 n+2}$ are the 
positions of the bottom node of the tether, and $\mathbf{p}=\left[\begin{array}{ll}x_{r} & y_{r}\end{array}\right]^{T}$ is the position vector of the centers of the rigid body.

The position vectors of the center of the rigid body is

$$
\mathbf{p}=\mathbf{R}-\mathbf{T r}=\left[\begin{array}{c}
e_{4 n+1} \\
e_{4 n+2}
\end{array}\right]-\left[\begin{array}{cc}
\cos \theta_{r} & -\sin \theta_{r} \\
\sin \theta_{r} & \cos \theta_{r}
\end{array}\right]\left[\begin{array}{c}
-d_{r} \\
0
\end{array}\right],
$$

where $\mathbf{T}$ is the transformation matrix and $d_{r}$ is the length between the center of gravity of a rigid body and the connecting position.

The constraint of the system is then described as

$$
\begin{aligned}
& \mathbf{C}(\mathbf{q})=\mathbf{p}-\mathbf{R}+\mathbf{T r} \\
& =\left[\begin{array}{l}
x_{\mathrm{r}}-e_{4 n+1}-d_{r} \cos \theta_{r} \\
y_{\mathrm{r}}-e_{4 n+2}-d_{r} \sin \theta_{r}
\end{array}\right]=0 .
\end{aligned}
$$

Using Lagrange multipliers, the equation of motion of the tethered system is

$$
\mathbf{M} \ddot{\mathbf{q}}+\mathbf{C}_{\mathbf{q}}^{T} \boldsymbol{\lambda}=\mathbf{Q} .
$$

Differentiating Eq. (16) twice with respect to time as

$$
\mathbf{C}_{\mathbf{q}} \ddot{\mathbf{q}}=-\left(\mathbf{C}_{\mathbf{q}} \dot{\mathbf{q}}\right)_{\mathbf{q}} \dot{\mathbf{q}}-2 \mathbf{C}_{\mathbf{q}} \dot{\mathbf{q}}-\mathbf{C}_{\mathbf{t t}} \equiv \boldsymbol{\gamma} \text {. }
$$

Using Eqs. (17) and (18), the differential algebraic equations can be obtained as

$$
\left[\begin{array}{cc}
\mathbf{M} & \mathbf{C}_{\mathbf{q}}^{\mathrm{T}} \\
\mathbf{C}_{\mathbf{q}} & \mathbf{0}
\end{array}\right]\left[\begin{array}{l}
\ddot{\mathbf{q}} \\
\lambda
\end{array}\right]=\left[\begin{array}{l}
\mathbf{Q} \\
\gamma
\end{array}\right],
$$

where $\mathbf{C}_{\mathbf{q}}$ is the following Jacobean matrix:

$$
\mathbf{C}_{\mathbf{q}}=\left[\begin{array}{cccccccc}
\cdots & -1 & 0 & 0 & 0 & 1 & 0 & d \sin \theta \\
\cdots & 0 & -1 & 0 & 0 & 0 & 1 & -d \cos \theta
\end{array}\right]
$$

3. Experiment for tethered system motion with large rotation, deformation and translation

There has been little experimental research on the motion with large deformation and displacement in a tethered system. Such experimental data would be helpful for evaluating a numerical approach. Therefore, a basic experiment on a tethered system was conducted in order to understand the motion of the tether and the coupled motion between the tether and the attached rigid body, and to obtain basic data to validate the proposed numerical approach for the motion of the tethered system. In the present paper, the case in which the mother ship moves as shown in Fig. 4 is assumed.

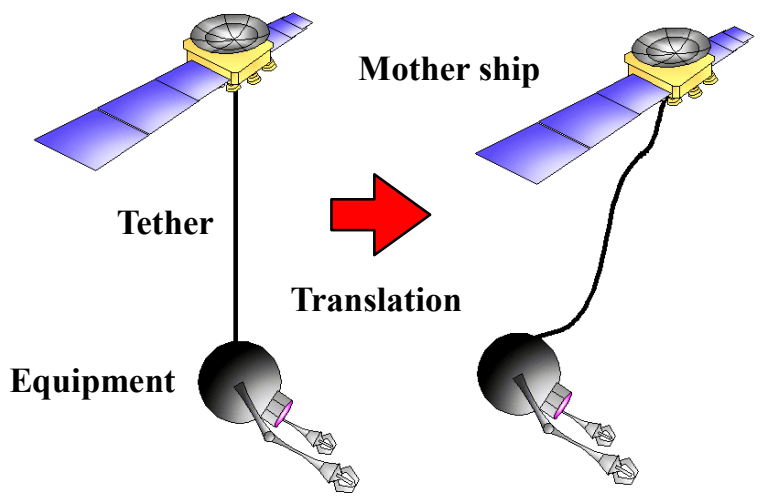

Fig. 4 Motion of a tethered system 


\subsection{Motion of bottom free tether using two types of materials}

In this section, the movement of one end of the tether is given, and large displacement and deformation of the tether occur. The present experiment was conducted in order to examine the basic motion of the tether. Steel and rubber were used as the materials because the physical properties of steel are known and rubber displays remarkable axial expansion and contraction properties.

In the present experiment, it is necessary to measure the tether motion using a non-contact method because the tether is light and the motion has large deformation and large displacement. Therefore, the tether is measured using digital video cameras. The image data measured by the two digital video cameras is visualized using software. The software can determine three-dimensional location information of an arbitrary point on the object body, based on the brightness information in the image data.

In the experimental device, the movement of one end of the tether is given by the movable object, as shown in Fig. 5. The top of the tether is fixed to the movable object by a rotation joint. The movable object controls the amount of straight advancement movement. A mass is attached at the bottom end of the tether. The joint between the movable object and the tether can rotate in two dimensions.

\section{1. 1. Stainless steel tether}

The purpose of this experiment is to investigate the motion of the tethered system with large deformation and displacement, using a stainless lamina as the model of the tether, and to obtain basic data to evaluate the validity of the numerical analysis. Even though this is a basic experiment, there are very few results available from other studies. It may be possible to use this experimental result to validate the results of modeling and formulation in flexible multibody dynamics.

Stainless steel SUS304 is used as the tether. This stainless lamina allows tether motion with large deformation. The reflective tape is pasted on the tether for the purpose of measurement. The parameters of the tether material and the attached mass are as follows: tether length; $1.02[\mathrm{~m}]$, density; $8.03 \times 10^{3}\left[\mathrm{~kg} / \mathrm{m}^{3}\right]$, sectional area; $1.6 \times 10^{-6}\left[\mathrm{~m}^{2}\right]$, thickness; $0.2 \times 10^{-3}[\mathrm{~m}]$, width; $8.0 \times 10^{-3}[\mathrm{~m}]$, modulus of elasticity; $1.97 \times 10^{11}\left[\mathrm{~N} / \mathrm{m}^{2}\right]$, and mass at the bottom end; $4.0 \times 10^{-2}[\mathrm{~kg}]$.

\section{1. 2. Rubber tether}

By using the rubber material, remarkable axial expansion and contraction are observed. A large deformation problem including these phenomena can be examined. In addition, rubber is useful because space is limited to a laboratory scale, and an actual tethered system is generally a large-scale system. In other words, measuring motion with large deformation is difficult because of the length limitation of the tether. Considering this limitation, an experiment using a tether with a low degree of elasticity is effective. The natural rubber which contains carbon is used as the tether. First of all, the expansion to a static load is measured with a spring scale because the measurement of the dynamic physical properties

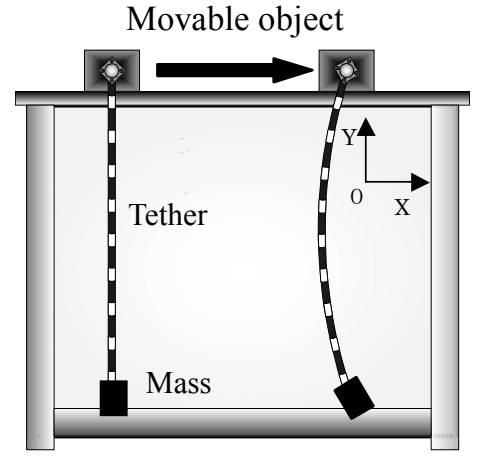

Fig. 5 Experimental Device

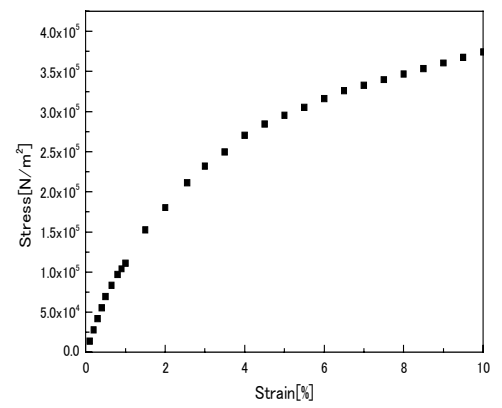

Fig. 6 Stress-Strain curve of the rubber material 
of the material is difficult. Figure 6 shows the stress-strain curve. For this material, the modulus of elasticity decreases as the strain increases. The parameters of the tether material and the mass at the bottom are as follows: tether length; $1.0[\mathrm{~m}]$, density; $0.014\left[\mathrm{~kg} / \mathrm{m}^{2}\right]$, sectional area of the tether; $7.069 \times 10^{-6}\left[\mathrm{~m}^{2}\right]$, diameter of the tether; $3.0 \times 10^{-3}[\mathrm{~m}]$, and mass at the bottom; $3.0 \times 10^{-3}[\mathrm{~kg}]$. In this experiment, two kinds of models, with the bottom mass and without bottom mass, are used. There are two purposes of this experiment. The first is to observe the coupling motion between the bottom mass and the tether, in which expansion and contraction are abundant. In this case, the ratio of the bottom mass and the mass of the tether is 1:3. Because the vibration phenomenon is strong and affects the longitudinal elasticity, the motion of the tether deviates from the planar motion. The second purpose is to examine the influence of the mass, that is, the influence of tension, on the behavior of the tether with the mass, as compared to that of the tether without the mass.

\subsection{Coupling motion between tether and attached rigid body under microgravity}

The experiment under microgravity is selected because the tether moves with large deformation and the rigid body moves with large rotation, even if the tension is small. Therefore, the coupling motion between the tether and the rigid body is excited. The purposes of this experiment are to observe the coupling motion between the tether and the rigid body and to obtain basic data by which to verify the numerical results of the numerical technique.

A drop experiment facility provides a good microgravity environment, which is obtained by freely dropping the capsule in a vacuum. This microgravity environment continues for approximately $4.5 \mathrm{sec}$. Figure 7 shows the fall tower and the fall capsule.

To conduct this experiment, the measuring method is first selected, and then the size of the experimental device is decided. It is necessary to place the observation object as well as the measurement and data logging equipment inside the capsule because the space for the experiment is completely isolated and limited. Wireless data transmitted from the capsule becomes unstable while the capsule falling. The measuring device is composed of two digital video cameras (DV), two CCD cameras.

The experimental device allows large deformation and large translation of the tethered system by recording the translation of the end plate moving at a constant speed. Figure 8 shows the experimental device, which consists of the moving end plate displacement device, the tethered system, and the rigid body support stand. The moving end plate displacement device was set to have a height of $0.6[\mathrm{~m}]$, a width of $0.15[\mathrm{~m}]$, and a depth of $0.1[\mathrm{~m}$ ]

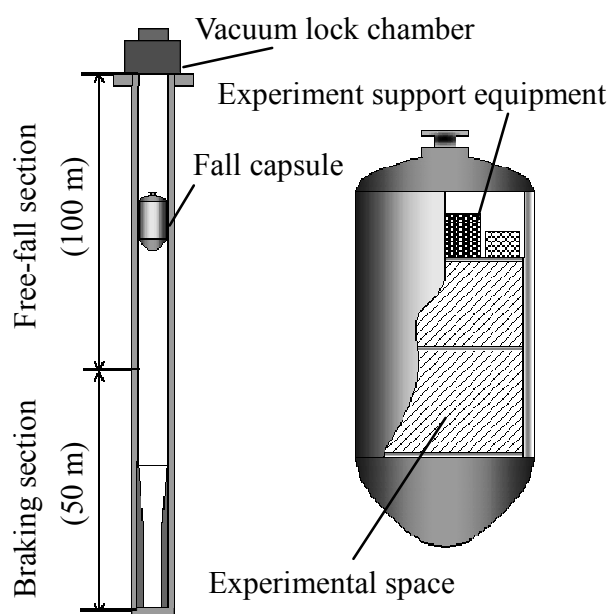

(a) Fall tower (b) Fall capsule

Fig. 7 Experimental device

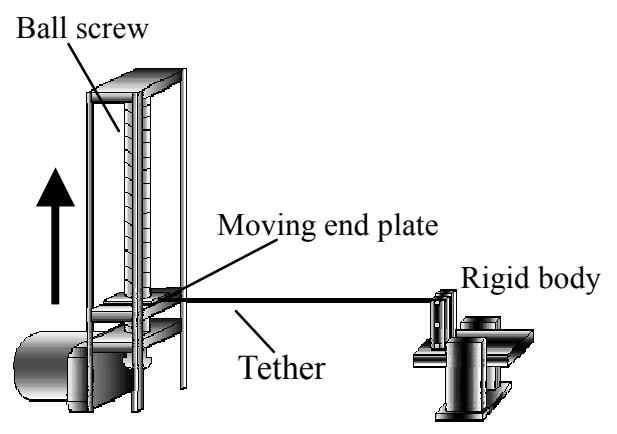

DC motor

Fig. 8 Experimental device 
because of the limited dimensions of the capsule. The rigid body support stand was necessary because the external force did not work a rigid body in the microgravity environment. The tether and the rigid body are connected to allow free rotation in two dimensions. The tether was fixed to the moving end plate, and the reflective tape was pasted on the tether for measurement. The size of this device is assumed to be the maximum in order to allow sufficient time for the tether motion with large displacement. The parameters of the tether and the rigid body are as follows: tether length; $0.26[\mathrm{~m}]$, mass of the rigid body; $0.12[\mathrm{~kg}]$, rigid body length; $0.05[\mathrm{~m}]$, and initial angle between the tether and the rigid body; $\pi / 2[\mathrm{rad}]$.

\section{Discussion of motion of tether and coupled system with rigid body}

In this section, the motion of the tethered system is examined numerically and experimentally. The movement of one end of the tether is given. The fundamental tether motion is discussed under a gravity environment. Two types of materials are used in order to discuss the effect of elasticity. The coupled motion between the tether and the rigid body is then investigated under microgravity.

\subsection{Fundamental motion of bottom free tether under movement of one end}

\section{1. 1. Stainless steel tether}

In this case, the tether is very flexible, although it is rigid with respect to axial deformation. The upper end of the tether is moved $0.55[\mathrm{~m}]$ at a velocity of $0.98[\mathrm{~m} / \mathrm{s}]$ except for a short period of acceleration. The number of elements is 50. In the initial stage, the tether is in a static equilibrium state. The experimental results of the variation of the shapes of the tether on the outward trip and on the return trip in 0.1-s intervals are shown in Fig. 9(a). The translation of the upper end causes lateral deformation of the tether. The deformation then increases with the movement. The deflection of the midpoint of the tether increases in the direction opposite to the direction of travel at $0.8 \mathrm{sec}$ to $1.0 \mathrm{sec}$. This phenomenon is caused by the coupling motion between the deflection of the tether and the inertia force of mass. The numerical results corresponding to the experimental result are shown in Fig. 9(b).

The experimental and numerical results are in good agreement, which confirms that the numerical approach with ANCF is efficient for problems with large rotation, deformation and translation. The time history of the lateral displacement of the midpoint of the tether during travel is also shown in Fig. 10. Thus, the numerical approach is effective for expressing the tether motion in detail.
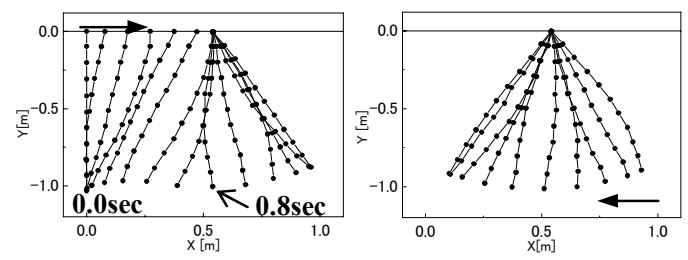

(a) Experimental
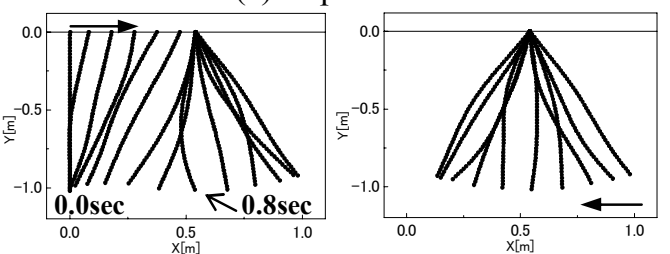

(b) Numerical

Fig. 9 Shape of the system during the outward trip and the return trip

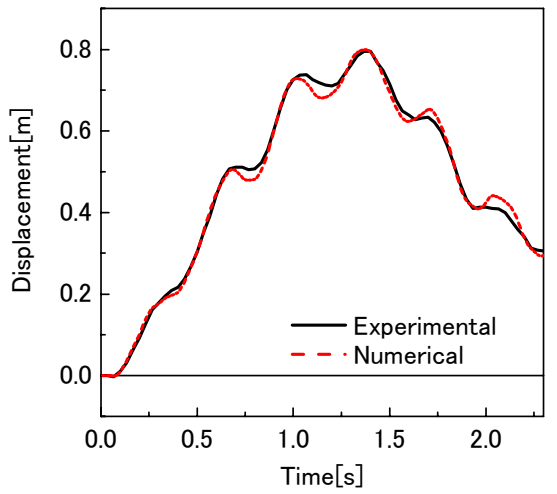

Fig. 10 Time history of the midpoint of the tether using a stainless steel tether 


\section{1. 2. Rubber tether}

In this experiment, the tether is made of rubber, and the axial elasticity during the motion of the tether is not negligible. The compulsion movement of the tether end as well as the preceding clause is given. The number of elements is 50. Two types of models are used in this experiment: a model with a bottom mass and a model without a bottom mass. The modulus of elasticity of linear range in Fig. 6 is used in the numerical simulation. The internal damping force is assumed to depend on the speed of expansion and contraction for the axial stress of the element. The experimental results of the variation of the shapes of the tether with a bottom mass on the outward and return trips in 0.1-s intervals are shown in Fig. 11(a). The difference of these results compared to the results for the steel tether is that the propagation speed of the transverse wave is very small, so the lateral oscillation is not great. Its mode shape is more complex than the shape of the steel tether. The numerical results are shown in Fig. 11(b). The numerical approach is also effective for determining the motion of this very flexible tether.

The experimental results of the variation of the shapes of the tether without a bottom mass on the outward and return trips in 0.1-s intervals are shown in Fig. 12(a). The difference between the results for the model with a bottom mass and the model with a bottom mass is obvious due to the shape of the tether at $1.2 \mathrm{sec}$. The shape of the tether at $1.2 \mathrm{sec}$ is generally observed in the case of a pendulum with large amplitude. Since the inertia of the bottom mass provides the tension in the tether and the deflection of the tether is small, this difference occurs. Therefore, the mass of the attached equipment has a significant influence on the motion of a tethered system with large deformation, even if the mass of the attached equipment is small. The numerical and experimental results are in good agreement. The time history of the lateral displacement of the midpoint of the tether is shown in Fig. 13, which compares the results with and without a bottom mass. Note that the

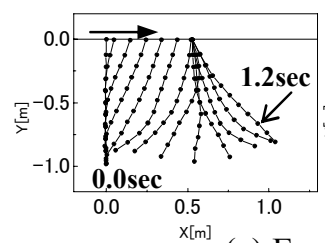

(a) Experimental
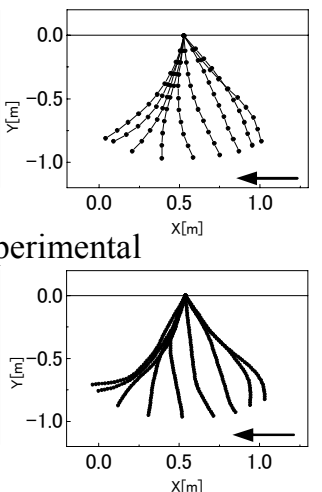

(b) Numerical

Fig. 11 Shape of the system with the bottom mass during the outward trip and the return trip
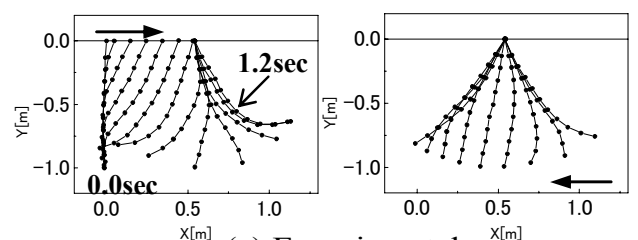

(a) Experimental
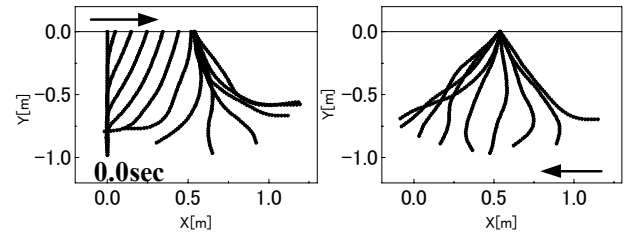

(b) Numerical

Fig. 12 Shape of the system without the bottom mass during the outward trip and the return trip

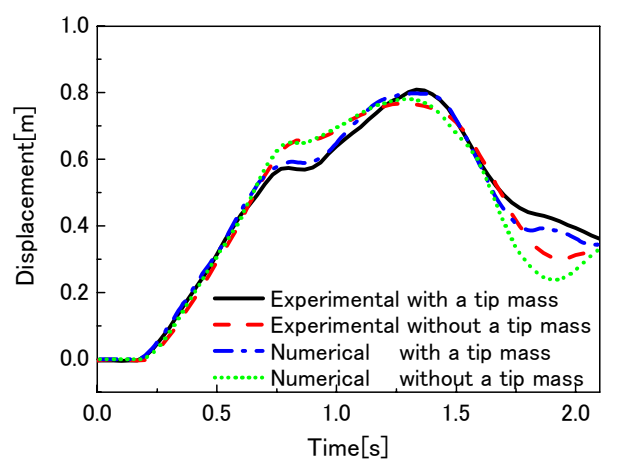

Fig. 13 Time history of the midpoint of the tether 
propagation of the transverse wave is slower in the system without a bottom mass. The system without a bottom mass has larger amplitude. These tendencies are found in both the numerical and experimental results.

\subsection{Coupling motion between tether and attached rigid body under microgravity}

The coupled motion between the tether and the attached rigid body is significant when its tension is small. Such a condition occurs in extreme environments such as in space and under deep sea. The purpose of this section is to observe the strongly coupled motion between the tether and the attached rigid body and to apply the proposed numerical approach in order to evaluate its validity. The corresponding experiment is discussed in Section 3 .

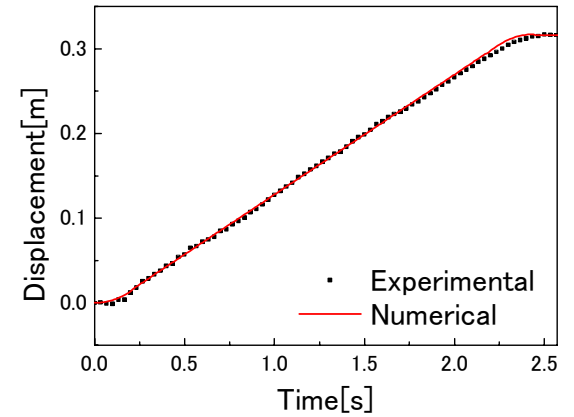

Fig. 14 Time history of the moving end of the tethered system

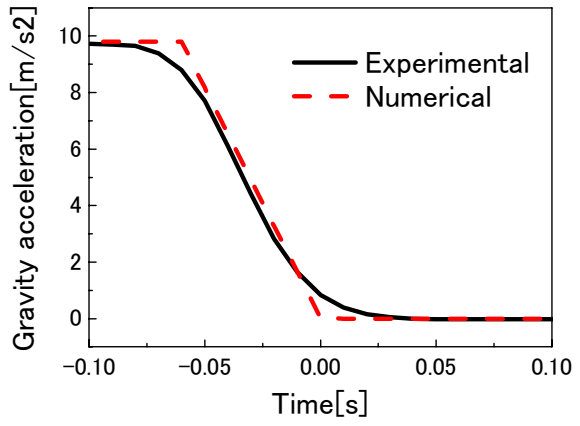

Fig. 15 Time history of gravity

The time history of the vertical movement of the tether end is shown in Fig. 14. The numerical analysis is shown by the solid line in this figure. The tether moves with a constant speed of $0.14[\mathrm{~m} / \mathrm{s}]$, except for a short period of acceleration. The time history of the acceleration of gravity in the experiment is shown in Fig. 15.

The experimental and numerical results in 0.2-s intervals for the tethered system of each shape are shown in Fig. 16. A qualitative coincidence is shown in these results. In the initial stage, the lateral deflection in the tether propagates. When the deflection of the tether reaches the end attached to the rigid body, the rigid body is given the moment by the tension acting on the connecting point. Then the rigid body translates with rotation.

During the period from $1.0 \mathrm{sec}$ to $1.6 \mathrm{sec}$, the rigid body translates without rotation,
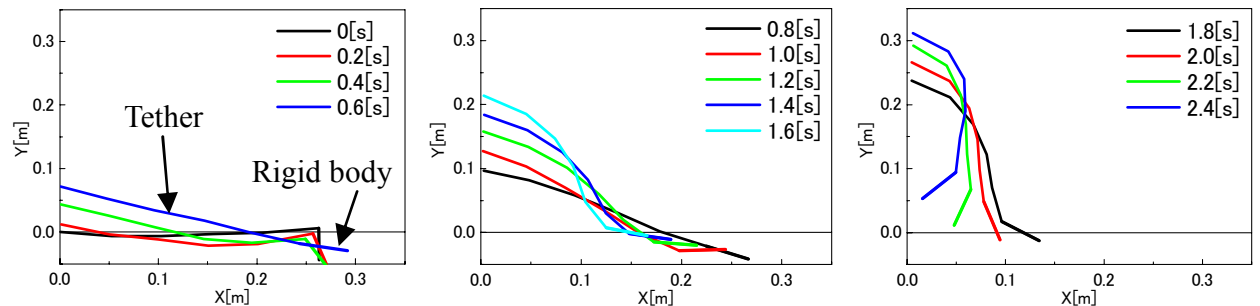

(a) Experimental
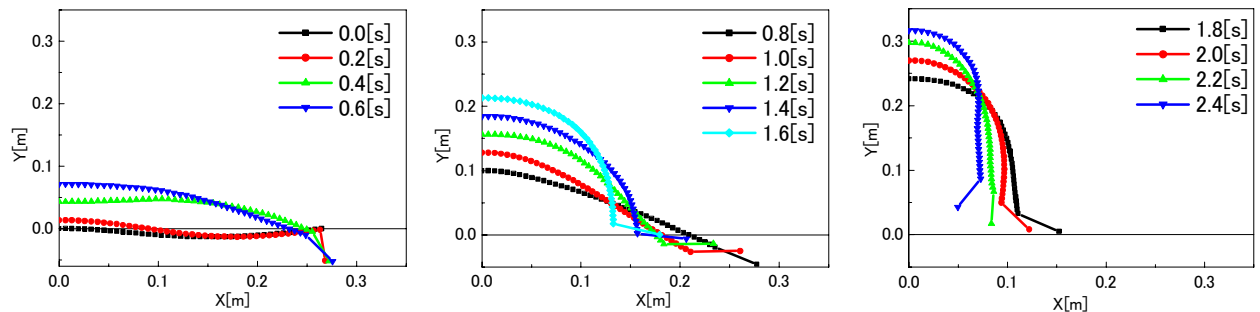

(b) Numerical

Fig. 16 Experimental results for the shape of the tethered system 


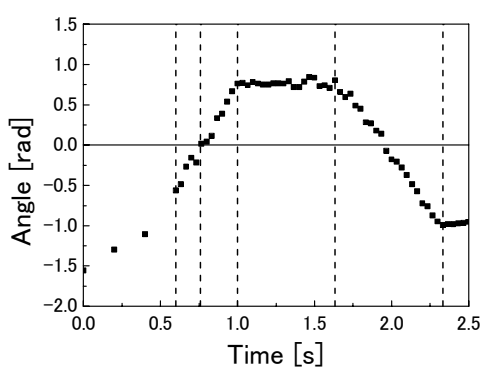

(a) Angle of rigid body

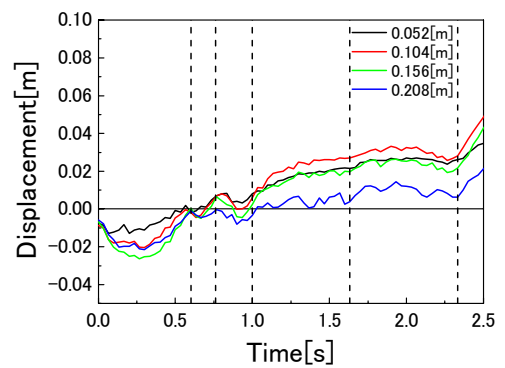

(b) Deflection of tether

Fig. 17 Time history of the tether motion (experimental)

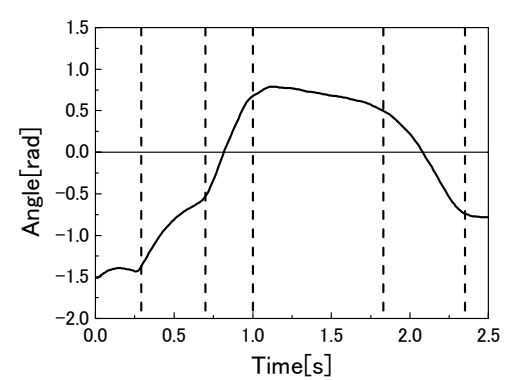

(a) Angle of rigid body

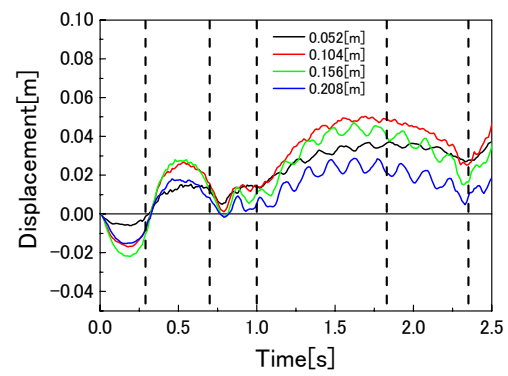

(b) Deflection of tether

Fig. 18 Time history of the tether motion (numerical)

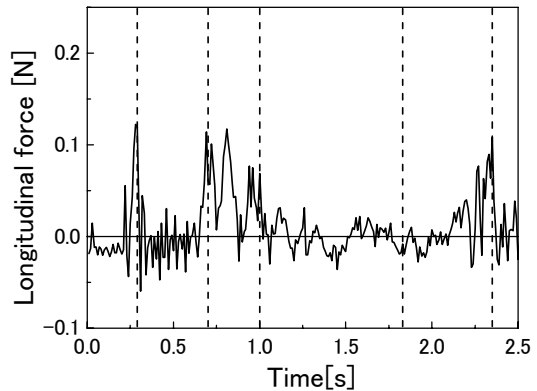

Fig. 19 Time history of the longitudinal force (numerical)

and then begins to rotate again at approximately $1.8 \mathrm{sec}$. To explain this phenomenon, the angle of the rigid body and the deflection in the direction normal to the axis that connects both ends of the tether are considered. The experimental results for the angle of the rigid body and the deflection at various positions on the tether are shown in Fig.s 17(a) and 17(b). The corresponding numerical results are shown in Fig.s 18(a) and 18(b). The five dotted lines in these figures show the time when the angle of the rigid body changes significantly. At these five points, the deflection of the tether becomes small. In other words, the tether has a straight shape, and the longitudinal force acts on the connecting point so that a moment is given to the rigid body. The numerical results also show this feature of the interaction between the tether motion and the rigid body rotation.

The numerical results for the time history of the longitudinal force of the tether at the connecting point are shown in Fig. 19. Note that the above discussion about the interaction is acceptable.

On the other hand, a reason for the significant change in the angle of the rigid body at $1.65 \mathrm{sec}$ is required. The tether deflection is not small at this stage. During the period from $1.0 \mathrm{sec}$ to $1.6 \mathrm{sec}$, the rigid body translates without rotation. This motion is caused by the relation between the given movement at the top end and the inertia force of the rigid body. Due to the movement at the top end, there is small longitudinal force in the tether. After this state continues for a while, a geometrical relation between the mode shape of the tether and the attitude of the rigid body excites the force acting on the connecting point between the tether and the rigid body again. 


\section{Summary}

Numerical and experimental approaches were applied to the motion of a tethered system with large rotation, deformation and translation. Modeling and formulation of the motion of a system consisting of a tether and a rigid body were proposed. The proposed modeling and formulation use the Absolute Nodal Coordinate Formulation and the equation of the constraint of the combination between flexible body motion and rigid body motion. We performed experiments on the motion of the tether and the tethered system under both gravity and microgravity environments.

The numerical approach for the tether motion with large rotation, deformation and translation using the Absolute Nodal Coordinate Formulation was verified to be effective, based on a comparison with the experimental results. Steel and rubber were used as the tether materials. From these results, characteristics of the motion of tether and tethered system are clarified. Moreover, the mechanism of the coupling between the tether motion and the rigid body motion under a microgravity environment was explained and the characteristics of the coupled motion were identified. Tether flexibility and influence of the attached equipment are an important factor for the tethered system in the coupling process. Furthermore, it was observed that the strong coupled motion between the tether and the rigid body increased under a microgravity environment.

The obtained experimental results were compared with the above-described numerical results. The numerical results were quantitatively and qualitatively in good agreement with the experimental results. The validity of the numerical formulation using ANCF for the tether and the tethered system was confirmed.

\section{Acknowledgments}

This research was supported in part by the Japan Space Forum.

\section{References}

(1) Modi, V. J., and Misra, A. K., 1979, "On the deployment dynamics of tether connected two-body systems," Acta Astronutica 6, pp. 1183-1197.

(2) Pradhan, S., Modi, V. J., and Misra, A. K., 1999, “Tether-Platform Coupled Control," Acta Astronutica 44, No. 5, pp. 243-256.

(3) Nohmi, M., and Yoshida, S., 2004, "Experimental Analysis for Attitude Control of a Tethered Space Robot under Microgravity," Space Technology 24, No. 2-3, pp. 119-128.

(4) Terumichi, Y., Ohtuka, M., Yoshizawa, M., Fukawa, Y., and Tsujioka, Y., 1997, "Nonstationary Vibrations of a Flexible body with Time-Varying length and a Mass-Spring System Attached at the Lower End," Nonlinear Dynamics 12, pp. 39-55.

(5) Kaczmarczyk, S., and Ostachowicz, W., 2000, "Non-Stationary Responses of Cables with Slowly Varying Length," J. Acoustics and Vibration., 5-3, pp. 117-126.

(6) Shabana, A. A., Hussien, H. A., and Escalona, J. L., 1998, "Application of the Absolute Nodal Coordinate Formulation to Large Rotation and Large Deformation Problems," ASME J. Mech. Des., 120, pp. 188-195.

(7) Berzeri, M., Campanelli, M., Shabana, A. A., 2001, "Definition of the Elastic Force in the Finite-Element Absolute Nodal Coordinate Formulation and the Floating Frame of Reference Formulation,” ASME J. Multibody Syst. Dyn., 5, pp. 21-54.

(8) Sugiyama, H., Mikkola, A. M., and Shabana, A. A., 2003, "A non-incremental nonlinear finite element solution for cable problems,” ASME J. Mech. Des., 125, pp. 746-756.

(9) Yoo, W. S., Lee, J. H., Sohn, J. H., Park, S. J., Dmitrochenko, O. N., Pogorelov, D. Y., 2003, "Large oscillation of a Thin Cantilever Beam: Physical Experiments and Simulation using Absolute Nodal Coordinate Formulation," Nonlinear Dynamics, 34(1), pp. 3-29. 